\title{
Current State and Future Prospects of Research on Fear of Cancer Recurrence
}

\author{
Sophie Lebel*1, Gozde Ozakinci ${ }^{2}$, Gerald Humphris ${ }^{2}$, Belinda Thewes ${ }^{3}$, Judith \\ Prins $^{3}$, Andreas Dinkel ${ }^{4}, \&$ Phyllis Butow ${ }^{5}$ \\ ${ }^{1}$ University of Ottawa \\ ${ }^{2}$ University of St Andrews \\ ${ }^{3}$ Radboud University Medical Centre \\ ${ }^{4}$ Technische Universität München \\ ${ }^{5}$ University of Sydney
}

\section{"Correspondence:}

University of Ottawa

136 Jean Jacques Lussier, room 4016

Ottawa, Canada

K1N6N5

(613) 562-5800, ext. 4811

slebel@uottawa.ca

\section{Acknowledgments:}

This work was supported by a Dissemination and Planning grant (\# PCS 142019) from the Canadian Institutes of Health Research and by a Meeting grant from the University of Ottawa to Sophie Lebel. We would like to thank all the Colloquium attendees for their contribution. 
1. Despite a rapidly growing research interest in FCR, lack of consensus on definition and measurement including clinical FCR, sparse model development and testing, and limited available clinical interventions have impeded knowledge transfer into patient services.

2. To move forward, a 2-day colloquium was held in Ottawa, Canada in August 2015 to progress knowledge and identify future research directions.

3. A comprehensive research program was proposed, including development of a clinical definition, an updated review of screening measures, and a review of existing interventions.

4. A new special interest group was created with the International Psychosocial Oncology Society (IPOS) to facilitate the implementation of this research program and future international collaborations. 
Fear of cancer recurrence (FCR) has been described as the sword of Damocles that hangs over survivors for the rest of their lives [1]. FCR is usually defined as "the fear or worry that the cancer will return or progress in the same organ or in another part of the body" [2,3]. FCR was the first or second most commonly reported problem by patients with breast, ovarian, colon, lung, or prostate cancer [4]. Overall, the current literature suggests that moderate to high levels of FCR, suggestive of clinical FCR, affect $49 \%$ of cancer patients [5] and that severe FCR tends to persist over time [6]. FCR is consistently associated with increased functioning impairment, psychological distress, stress-response symptoms, and lower quality of life [5-7], yet is distinct from other anxiety disorders [8]. High FCR is also associated with greater utilization of healthcare resources, thus suggesting that untreated FCR is likely to increase cost to the healthcare system [9].

Despite clear evidence that cancer patients with higher FCR have higher psychological morbidity and may incur additional medical costs, we believe there are numerous gaps in the current literature that need addressing for the field of FCR research and intervention to progress. These include reaching consensus on 1) definitions of FCR and clinical FCR; 2) whether to and how to screen for clinical FCR; 3) which measures to use in the clinical setting; 4) which model(s) to use to guide interventions, 5) and how to manage this problem clinically. To start addressing these gaps and propose a coherent research agenda, a two-day Colloquium on FCR was held in Ottawa, Canada, on August 3 and $4^{\text {th }}, 2015$ to bring together international researchers, policy makers, and patient advocates. See Table 1 for a summary of objectives and the proposed research program emerging from the meeting.

\section{Objective 1: Agreeing on a definition of FCR and on the diagnostic characteristics of clinical FCR}

While the above definition is often used, there is no consensus among researchers that it adequately describes the phenomenon of FCR. For example, this definition does not reflect that FCR is a normal reaction to cancer. Also, the existing definition of FCR does not fit well with recent advances in cancer medicine. Therapies may be ongoing for many years and cancer is more and more seen as a chronic illness. As such, attendees of the FCR colloquium reviewed currently used definitions for accuracy and completeness and reached a consensus on a new definition of FCR using a Delphi study method [Lebel et al. Submitted].

Also, while there is consensus that FCR ranges from "normal" to "clinical", there is no agreement about what constitutes a clinical level of FCR. Discussion between researchers and clinicians is required to enable agreement on the diagnostic characteristics of clinical FCR. Attendees discussed the consequences of diagnostic labelling. While it was recognised that a clinical diagnosis may be stigmatising, attendees felt a clinical cut-off would: (i) instigate effort to assist patients with higher than average levels of FCR, (ii) prevent unnecessary use of limited resources and (iii) focus research activity to those with problematic recurrence fears. Attendees identified elements of clinical FCR based on their clinical experience and the existing literature [Lebel et al. Submitted]. These elements will be refined in a second Delphi study.

\section{Objective 2: Measuring clinical FCR}


The absence of an agreed upon definition of clinical FCR has made the assessment of this phenomenon challenging. Studies that attempt to define clinical FCR, such as the proposed Delphi study, will draw together informed opinion to arrive at a best fitting solution. Such a development would facilitate efforts to create measures of 'clinical' FCR comprising: (i) self-report questionnaires and/or (ii) standardised interview procedures. It will be important for such measures to specify: the formality of the procedure, the assessor, timing (e.g., first follow-up and frequency of repeat assessment), setting conditions (e.g., oncology clinic), length, complexity, and measurement conceptual structure.

Some FCR instruments have cut-off scores to identify "high" or "clinical" FCR [5, 10], but the lack of a gold-standard clinical interview or measure makes validating cut-offs difficult. A clinical interview was developed and used to validate the cut-off score of the Fear of Cancer Recurrence Inventory-Short Form [11] but we need to further validate its content and consider modifying it to reflect agreed upon definitions of FCR and clinical FCR. A gold-standard interview to detect clinical FCR is essential to assess the sensitivity and specificity of available FCR measures at detecting clinical FCR.

\section{Objective 3: Creating guidelines in the use and implementation of FCR measures in a clinical setting}

There are over 30 FCR measures available to researchers and clinicians [12]. While some instruments have been recommended for clinical and research use based on available evidence of their strong psychometric properties, they are currently not being routinely used by oncology centers to assess FCR among their patients.

The next step is the development and validation of short screening measures that can be used in clinical settings. There are newly-developed brief measures which may prove promising for FCR screening, validated amongst colorectal [13], breast [10,14], prostate [vd Wal et al. Unpublished] and head and neck cancer survivors [15]. We need studies that assess how we can incorporate such measures into routine screening efforts. Additional research priorities for this topic area are summarized in Table 1.

\section{Objective 4: Elaborating and/or refining theoretical models}

There is a growing body of research on predictors and correlates of FCR, as evidenced by the recent publication of three reviews of the literature on this topic [5-7]. However, while more than 40 predictors of FCR have been studied [6], few have been identified as "strong" predictors of FCR. The exceptions are younger age, which is not amenable to change, and presence of somatic symptoms. These disparate findings can be explained by the lack of available models of FCR. Until recently, there was only one proposed model of FCR [16], which has not yet been empirically validated. Newer therapeutic models have been proposed $[17,18]$ and could be used to guide model studies. A research priority is to identify the basic common elements of therapeutic FCR models and start empirically validating their proposed relationship with FCR. For example, the first results on metacognitions and implicit processes show that these may be promising mechanisms to consider [19].

\section{Objective 5: Next steps for interventions}


Despite the high prevalence of FCR and its impact on the patient and the health care system, a recent study showed that oncology specialists refer only $21 \%$ of patients with high FCR for psychosocial services and are unsure about the best approach to treat this common problem [20]. Fortunately, several interventions are currently being tested in randomized controlled trials, which hopefully will lead to evidence based guidelines on how to manage FCR in the near future. These are specifically designed to alleviate clinical or moderate to high FCR, and are largely based on a cognitive-behavioral model of psychotherapy. However, each intervention includes unique ingredients (e.g. meta-cognitive therapy or exposure) and format of delivery (e.g. group, blended care, individual). At this point, research is needed to identify the active ingredients in current FCR interventions. Additional research priorities within this topic are summarized in Table 1.

\section{Objective 6: Formally structuring researchers interested in FCR as a research group}

To ensure continued optimal development of this research area, a Special Interest Group under the banner of the International Psycho-Oncology Society, called FORwaRdS (the Fear Of RecuRrence Special interest group) was created.

\section{CONCLUSION}

Several concrete projects are planned following an expert and stakeholders meeting to address gaps in the literature on FCR. The proposed program of research will hopefully lead to guidelines being available in the near future to orient clinical care for cancer patients struggling with FCR. 
Table 1. Proposed program of FCR research

\begin{tabular}{|c|c|}
\hline Objective & Future research steps and recommendations \\
\hline $\begin{array}{l}\text { Agreeing on a } \\
\text { definition of FCR and } \\
\text { on the diagnostic } \\
\text { characteristics of } \\
\text { clinical FCR }\end{array}$ & $\begin{array}{l}\text { Publish the new definition of FCR and the elements of clinical FCR } \\
\text { identified during the Colloquium }\end{array}$ \\
\hline Measuring clinical FCR & $\begin{array}{l}\text { - The elements identified to describe clinical FCR will be submitted } \\
\text { to researchers and clinicians using a formal Delphi survey } \\
\text { - Following the results of this survey, a 'gold standard' interview will } \\
\text { be proposed and tested }\end{array}$ \\
\hline $\begin{array}{l}\text { Creating guidelines in } \\
\text { the use and } \\
\text { implementation of } \\
\text { FCR measures in a } \\
\text { clinical setting }\end{array}$ & $\begin{array}{l}\text { - An updated systematic review of FCR measures focussing on } \\
\text { recommendations about measures to use for screening, case- } \\
\text { finding and intervention purposes will be written up } \\
\text { Developing cross-culturally valid short screening instruments and } \\
\text { longer instruments that reflect definitions of FCR and clinical FCR } \\
\text { across diverse range of respondents (including carers), cultures, } \\
\text { and languages is a priority }\end{array}$ \\
\hline $\begin{array}{l}\text { Elaborating and/or } \\
\text { refining theoretical } \\
\text { models }\end{array}$ & $\begin{array}{l}\text { - Testing (segments of) models and developing experimental } \\
\text { paradigms are a priority }\end{array}$ \\
\hline $\begin{array}{l}\text { Next steps for } \\
\text { interventions }\end{array}$ & $\begin{array}{l}\text { - A literature review on existing interventions will be written up } \\
\text { - The following are identified as priorities: } \\
\circ \quad \text { Testing if tailored interventions are more effective than } \\
\text { generic interventions at addressing FCR } \\
\circ \quad \text { Studying the effect of couple- and family-focused } \\
\text { interventions on FCR } \\
\circ \quad \text { Evaluating possible iatrogenic effects of FCR interventions }\end{array}$ \\
\hline $\begin{array}{l}\text { Formally structuring } \\
\text { researchers interested } \\
\text { in FCR as a research } \\
\text { group }\end{array}$ & $\begin{array}{l}\text { - Forming a new special interest group as part of International } \\
\text { Psycho-Oncology Society }\end{array}$ \\
\hline
\end{tabular}


References

1. Muzzin L, Anderson NJ, Figueredo AT, Gudelis SO. The experience of cancer. Social Science and Medicine 1994; 38(9):1201-1208.

2. Vickberg SM. The Concerns About Recurrence Scale (CARS): A systematic measure of women's fears about the possibility of breast cancer recurrence. Annals of Behavioral Medicine 2003; 25: 16-24.

3. Simard S, Savard J. Fear of Cancer Recurrence Inventory: Development and initial validation of a multidimensional measure of fear of cancer recurrence. Supportive Care in Cancer 2009; 17(3): 241-51.

4. Baker F, Denniston M, Smith T, West MM. Adult cancer survivors: How are they faring? Cancer 2005; 104(S11):2565-76.

5. Simard S, Thewes B, Humphris G, Dixon M, Hayden C, Mireskandari S, Ozakinci G. Fear of cancer recurrence in adult cancer survivors: A systematic review of quantitative studies. Journal of Cancer Survivorship 2013; 7(3): 300-322.

6. Crist JV, Grunfeld EA. Factors reported to influence fear of recurrence in cancer patients: a systematic review. Psycho-Oncology 2013; 22(5):978-86.

7. Koch L, Jansen L, Brenner H, Arndt V. Fear of recurrence and disease progression in long-term ( $\geq 5$ years) cancer survivors - a systematic review of quantitative studies. Psycho-Oncology 2013; 22(1):1-11.

8. Dinkel A, Kremsreiter K, Marten-Mittag B, Lahmann C. Comorbidity of fear of progression and anxiety disorders in cancer patients. General Hospital Psychiatry 2014; 36(6): 613-619.

9. Lebel S, Tomei C, Feldstain A, Beattie S, McCallum M. Does fear of cancer recurrence predict cancer survivors' health care use? Supportive Care in Cancer 2013; 21(3):901-6.

10. Thewes B, Zachariae R, Christensen S, Nielsen T, Butow P. The Concerns About Recurrence Questionnaire: validation of a brief measure of fear of cancer recurrence amongst Danish and Australian breast cancer survivors. Journal of Cancer Survivorship 2015; 9(1):68-79.

11. Simard S, Savard J. Screening and comorbidity of clinical levels of fear of cancer recurrence. Journal of Cancer Survivorship 2015; 9(3):481-91.

12. Thewes B, Butow $P$, Zachariae R, Christensen S, Simard S, Gotay C. Fear of cancer recurrence: a systematic literature review of self-report measures. Psycho-Oncology 2012; 21(6):571-87.

13. Custers JAE, Gielissen MFM, Janssen SHV, De Wilt JHW, Prins JB. Fear of Cancer Recurrence in Patients with Colorectal Cancer. Supportive Care in Cancer 2015; Epub ahead of print.

14. Custers JAE, van den Berg SW, van Laarhoven HWM, Bleiker EMA, Gielissen MFM, Prins JB. Cancer Worry Scale as instrument for detecting Fear of Recurrence in breast cancer survivors. Cancer Nursing 2014; 37(1): 44-50.

15. Ghazali N, Cadwallader E, Lowe D, Humphris G, Ozakinci G, Rogers SN. Fear of recurrence among head and neck cancer survivors: longitudinal trends. Psycho-Oncology 2013; 22(4): 807-13.

16. Lee-Jones C, Humphris G, Dixon R, Hatcher MB. Fear of cancer recurrence--A literature review and proposed cognitive formulation to explain exacerbation of recurrence fears. PsychoOncology 1997; 6(2):95-105. 
17. Butow P, Bell ML, Smith A B, Fardell JE, Thewes B, Turner J, Gilchrist J, Beith J, Girgis A, Sharpe L, Shih $S$, Mihalopolous $C$, members of the Conquer Fear Authorship Group. Conquer fear: protocol of a randomised controlled trial of a psychological intervention to reduce fear of cancer recurrence. BMC Cancer 2013; 13:201. doi:10.1186/1471-2407-13-201

18. Lebel S, Maheu C, Lefebvre M, Secord S, Courbasson C, Singh M, Jolicoeur L, Banea A, Harris C, Fing Kee Fung M, Rosberger Z, Catton P. Addressing fear of cancer recurrence among women with cancer: A pilot study of a 6-week group cognitive-existential intervention. Journal of Cancer Survivorship 2014; 8(3):485-96.

19. Butow P, Kelly S, Thewes B, Hruby G, Sharpe L, Beith J. Attentional bias and metacognitions in cancer survivors with high fear of cancer recurrence. Psycho-Oncology 2015. 24(4): 416-423.

20. Thewes B, Brebach R, Dzidowska M, Rhodes P, Sharpe L, Butow P. Current approaches to managing fear of cancer recurrence; a descriptive survey of psychosocial and clinical health professionals. Psycho-Oncology 2014; 23(4):390-6. 